\title{
Exploring Temperature-Modulated Operation Mode of Metal Oxide Gas Sensors for Robust Signal Processing ${ }^{+}$
}

\author{
Romana Boiger *, Stefan Defregger, Mirza Grbic, Anton Köck, Manfred Mücke, \\ Robert Wimmer-Teubenbacher and Bernat Zaragoza Travieso
}

Materials Center Leoben Forschung GmbH, Roseggerstraße 12, 8700 Leoben, Austria; stefan.defregger@mcl.at (S.D.); mirza.grbic@mcl.at (M.G.); anton.koeck@mcl.at (A.K.); manfred.muecke@mcl.at (M.M.); robert.wimmer-teubenbacher@mcl.at (R.W.-T.);

bernat.zaragoza-travieso@mcl.at (B.Z.T.)

* Correspondence: romana.boiger@mcl.at; Tel.: +43-3842-45922-539

† Presented at the Eurosensors 2018 Conference, Graz, Austria, 9-12 September 2018.

Published: 13 February 2019

\begin{abstract}
Metal oxide (MOx) gas sensor signals are mainly governed by adsorption and desorption processes of oxygen and its reaction with surrounding gas molecules. Different target gases exhibit different reaction rates leading to characteristic sensor responses for specific gas species and their concentrations. In this work, we compare temperature-modulated sensor operation (TMO) with sensor operation at a single temperature. Further, we explore if under specific TMO regimes, a simple signal processing allows for quantification of gas concentrations. We specifically investigate, if the relevant information can be captured in selected discrete wavelet coefficients. In addition, we compare the results received from this wavelet features to reaction rate evaluation features.
\end{abstract}

Keywords: metal oxide gas sensors; temperature-modulated operation; wavelet transform; gas concentration quantification

\section{Introduction}

Metal oxide (MOx) gas sensors have a wide area of application, ranging from indoor air quality control to environmental monitoring. The benefits arise from their low costs, low power consumption, small size, high material sensitivity and quick response [1]. Conventional conductometric MOx gas sensor devices use MEMS-based microheaters to enable operation temperatures up to $500{ }^{\circ} \mathrm{C}$. MOx gas sensors can be operated at constant temperatures or by temperature modulation. Hereby the microheaters enable fast heating and cooling ramps.

The gas-specific reaction kinetics are based on the interaction of oxygen, adsorbed on the surface of the sensing material, and the test gas. The reaction kinetic can be influenced by different temperatures. Different sensors, together with TMO and machine learning algorithms are able to identify gases and quantify gas concentrations. This paper has two goals, on the one hand it compares different TMO modes to enable more stable gas measurement signal processing. On the other hand it presents and compares features derived from gas sensor responses to specific TMO modes. These features can then be further used for gas identification and quantification in machine learning algorithms.

The paper is structured as follows: Section 2 describes the measurement setup and three different experiments being conducted. Section 3 compares measurements of these three different operation modes. Section 4 depicts methods for feature calculation. In Section 5 the features defined in Section 
4 are computed and presented for a specific TMO mode to distinguish different gas concentrations of test gas $\mathrm{CO}$ and is followed by a conclusion in Section 6 .

\section{Experimental Setup and MeasurementDescription}

Gas sensor measurements were performed at the gas measurement setup at Materials Center Leoben (MCL), consisting of several mass flow controllers, a relative humidity sensor and a Keithley 3706A Switch. The setup ensures a controlled environment and a reliable resistance measurement of the discrete gas sensors. An additional in-house python software allows automatic measurements. The software operates the gas control and the switch according to the given experiment definition files and merges all the measurement data of an experiment into one hdf5 file. The experiment definition file specifies the test gases (e.g., $\mathrm{CO}$ ) with the according concentrations, the relative humidity level, the heater temperature and the duration of each temperature step. The background gas for all the measurements is synthetic air $\left(80 \% \mathrm{~N}_{2}, 20 \% \mathrm{O}_{2}\right)$. Summarized, the measurement setup allows gas pulse measurements as well as TMO in an automated process. For this paper we used 2 different kinds of experiments for a CO sensor (AS-MLC, ams Sensors Germany GmbH). The temperature profiles can be seen in Figure 1 and the experiments were conducted as follows: Experiment 1 (Exp1): 3 different temperature profiles ((i) constant temperature $\left(450^{\circ} \mathrm{C}\right.$ ), (ii) two fast changing temperature steps from $450^{\circ} \mathrm{C}(0.4 \mathrm{~s})$ to $150^{\circ} \mathrm{C}(0.2 \mathrm{~s})$, (iii) several fast changing temperature steps from $450{ }^{\circ} \mathrm{C}(0.4 \mathrm{~s})$ to $200-440{ }^{\circ} \mathrm{C}$, resp., increasing always by $20^{\circ} \mathrm{C}(0.2 \mathrm{~s})$ and repeating it), lasting for total $410 \mathrm{~s}$ at $50 \%$ relative humidity in synthetic air were compared.

Experiment 2 (Exp2): The TMO of Exp1 (iii) was used, followed by 3 additional temperature jumps-from $450{ }^{\circ} \mathrm{C} \mathrm{(3} \mathrm{s)} \mathrm{to} 150{ }^{\circ} \mathrm{C}, 200{ }^{\circ} \mathrm{C}, 250{ }^{\circ} \mathrm{C}$ resp. $(26 \mathrm{~s})$ at $50 \%$ relative humidity in synthetic air with different concentrations of $\mathrm{CO}$ as test gas $(0,1,3,5,7,10,12,14,15,16,18,20,25,30,40,50$, $100 \mathrm{ppm})$.

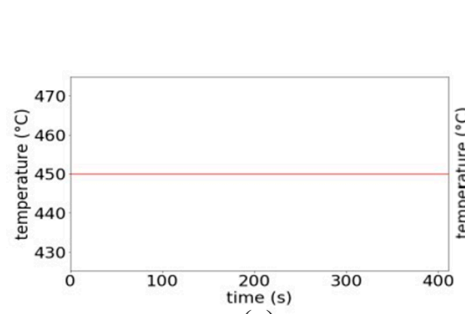

(a)

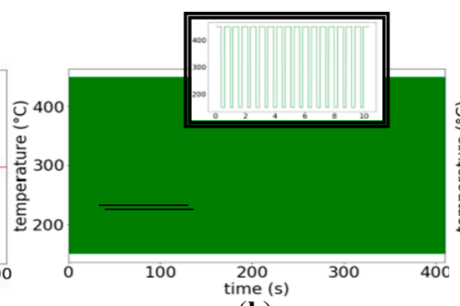

(b)

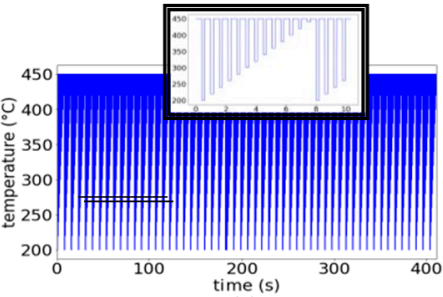

(c)

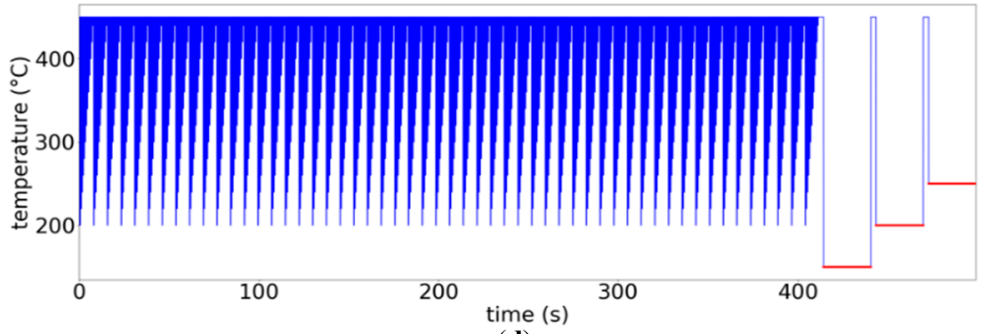

(d)

Figure 1. Temperature profiles for: (a) Exp1 (i); (b) Exp1 (ii); (c) Exp1 (iii); (d) Exp2.

\section{Results Experiment Exp1: Single Temperature Operation Versus TMO Mode}

The goal of Exp1 is to compare single temperature operation and temperature modulated operation. When a gas sensor is operated at a constant temperature without test gas, an equilibrium between adsorption and desorption of oxygen species is reached after some time [2], which can be seen by a nearly constant gas sensor resistance value $(R)$. Exp1 showed that such an equilibrium can be reached faster by TMO measurements, as can be seen in Figure 2a, where the logarithm of the conductance $\left(\ln G=\ln \frac{1}{R}\right)$ is depicted as a function of time $\ln G(t)$. Comparing the curves formed by the local maxima of $\ln G(t)$ of each temperature jump in Exp1(ii), (iii) with the response curve $\ln G(t)$ of Exp1(i), the following observation can be made. In Exp1(i) it lasts approx. 200 s until ln $G$ 
reaches $98 \%$ of $\ln G(410)$, the last measured value of the experiment. Whereas for the other two experiments Exp1(ii), (iii) the $98 \%$ of the last value are already reached after approx. 15, $17 \mathrm{~s}$, resp. This means that already after a very short time period the $\ln G$ values are not changing much anymore, hence measurement time until stabilization could be reduced by using TMO mode, compared to single temperature operation.

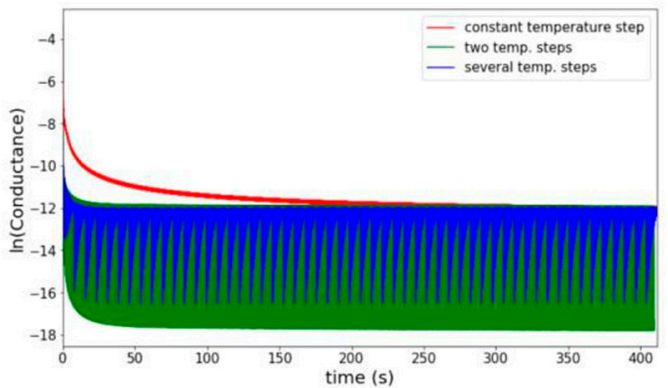

(a)

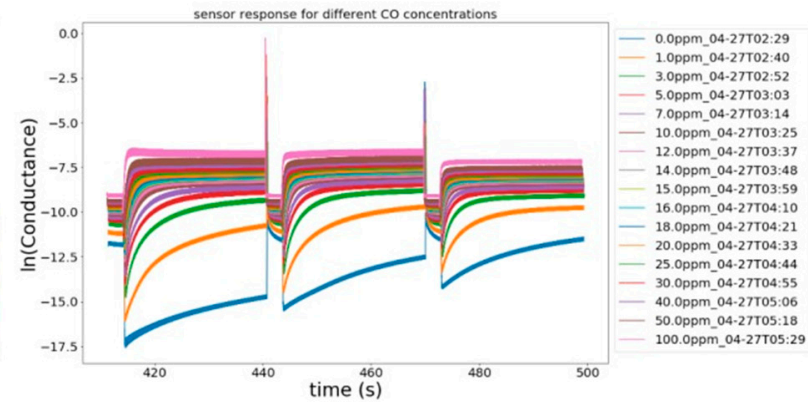

(b)

Figure 2. (a) Response curves $(\ln (G))$ to different TMO modes-Exp1 (i)-(iii); (b) Response curves $(\ln (G))$ to the three temperature jumps for different concentrations of $\mathrm{CO}-\mathrm{Exp} 2$.

\section{Feature Extraction Methods}

Features extracted from measurement data are important for using machine learning algorithms to detect and quantify gases [3]. As Schultealbert et al. [4,5] point out, for gas sensors, such features should be extracted from the response of the sensor at a low-temperature level, following a jump from a higher temperature level. Such jumps are present in experiment Exp2 and the considered lowtemperature levels are marked in red in Figure 1d.

\subsection{Features Extracted from Curve Fitting Parameters: $\tilde{k}$ and $\tau$ Evaluation}

In [5] two different methods for feature calculation are proposed. For low gas concentrations, where the sensor response has not yet approached an equilibrium, they suggest to use the $\tilde{k}$ evaluation. Hereby, $\tilde{k}$ is the slope of $\ln (G(t))$, which is an estimation of the reaction rate constant. For higher gas concentrations, where the sensor response has already stabilized the $\tau$ evaluation is applied. In [5] the time-constant $\tau$ of the relaxation process is defined as $\tau=t\left[\left(1-\frac{1}{e}\right)\left(\ln G_{\max }-\ln G_{\min )}\right]-\mathrm{t}_{\text {start }}\right.$ and $\frac{1}{\tau}$ is proposed to be used as a feature.

\subsection{Features Extracted from Transform Domain: Wavelet Coefficients}

In comparison to the features extracted from curve fitting parameters, where some knowledge about the curve itself is presumed, wavelet coefficients do not assume any specific curve form [6]. A wavelet transform uses basis functions to map the original signal from time into frequency space.

For the computation of the wavelet coefficients the python module PyWavelets (pywt.dwt with wavelet $=$ 'db4' and mode $=$ 'smooth') is used.

\section{Results Exp2: Comparison of Features for GasQuantification}

The three types of features introduced in Chapter 4 were computed for the sensor responses of Exp2 to different concentrations of $\mathrm{CO}$ to show the possibilities of gas quantification. The features plotted versus the concentration are depicted in Figure 3. The $\tilde{k}$ evaluation shows good results for lower concentrations, whereas the concentration distinction gets harder for higher gas concentrations, because the $\tilde{k}$ values are getting closer. The $\tau$ evaluation for this experiment seems to perform good also for lower concentrations, in contrast to the observations in [5]. The wavelet 
features, in this case the first wavelet approximation coefficient is used, however, show a comparable result to the $\tau$ and $\tilde{k} \quad$ evaluation for low and high gas concentrations.

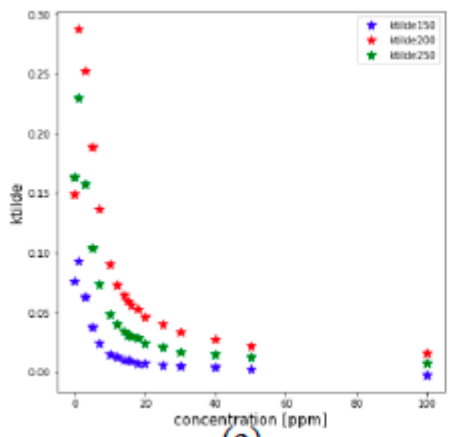

(a)

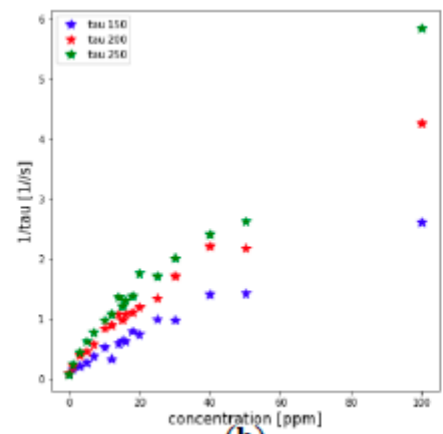

(b)

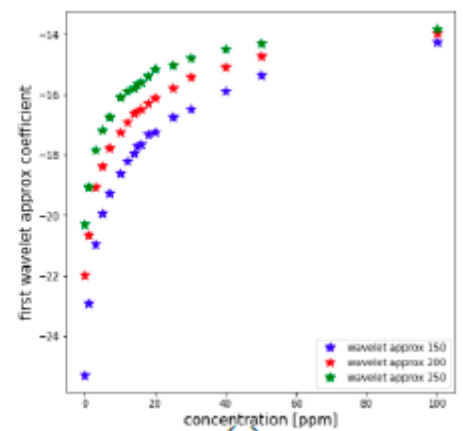

$(c)$

Figure 3. Features plotted versus various CO concentrations (a) Feature: $\tilde{k}$; (b) Feature: $1 / \tau$; (c) Feature: first wavelet approximation coefficient.

\section{Conclusions}

We compared TMO gas sensor measurements with sensor operation at a single temperature. Our experiments showed that the sensor response stabilized faster with TMO, hence TMO can be used to decrease the sensor operation time. With the second experiment we showed that features extracted from curve fitting parameters as well as features extracted from transform domain are suited for gas quantification with a single sensor. In further research this study should be extended to address the applicability of the features for gas identification for a single sensor. Furthermore, it would be interesting to investigate whether an array of gas sensors in combination with the above features can improve the gas identification and gas concentration quantification.

Author Contributions: R.B., S.D. and R.W.T. conceived and designed the experiments; B.Z.T. and M.G. programmed the measurement software and performed the experiments; R.B., S.D., M.M. and R.W.T. analyzed the data; A.K. and R.W.T. contributed materials and measurement tools; R.B. wrote the paper.

Acknowledgments: This work is done as part of the ECSEL project "IoSense-Flexible FE/BE Sensor Pilot Line for the Internet of Everything" (Grant Agreement No. 692480, FFG Project No. 6054961). Sensor material under investigation was provided by ams Sensors Germany GmbH.

Conflicts of Interest: The authors declare no conflict of interest. The founding sponsors had no role in the design of the study; in the collection, analyses, or interpretation of data; in the writing of the manuscript, and in the decision to publish the results.

\section{References}

1. Fine, G.F.; Cavanagh, L.M.; Afonja, A.; Binions, R. Metal Oxide Semi-Conductor Gas Sensors in Environmental Monitoring. Sensors 2010, 10, 5469-5502, doi:10.3390/s100605469.

2 Ding, J.; McAvoy, T.J.; Cavicchi, R.E.; Semancik, S. Surface state trapping models for $\mathrm{SnO}_{2}$-based microhotplate sensors. Sens. Actuators B 2001, 77, 597-613, doi:10.1016/S0925-4005(01)00765-1.

3. Yan, J.; Guo, X.; Duan, S.; Jia, P.; Wang, L.; Peng, C.; Zhang, S. Electronic Nose Feature Extraction Methods: A Review. Sensors 2015, 15, 27804-27831, doi:10.3390/s151127804.

4. Schultealbert, C.; Baur, T.; Schütze, A.; Böttcher, S.; Sauerwald, T. A novel approach towards calibrated measurement of trace gases using metal oxide semiconductor sensors. Sens. Actuators B 2017, 239, 390-396, doi:10.1016/j.snb.2016.08.002. 
5. Schultealbert, C.; Baur, T.; Schütze, A.; Sauerwald, T. Facile Quantification and Identification Techniques for Reducing Gases over a Wide Concentration Range Using a MOS Sensor in Temperature-Cycled Operation. Sensors 2018, 18, 744, doi:10.3390/s18030744.

6. Llobet, E.; Brezmes, J.; Ionescu, R.; Vilanova, X.; Al-Khalifa, S.; Gardner, J.W.; Bârsan, N.; Correig, X. Wavelet transform and fuzzy ARTMAP-based pattern recognition for fast gas identification using a microhotplate gas sensor. Sens. Actuators B 2002, 83, 238-244, doi:10.1016/S0925-4005(01)01047-4.

(C) 2019 by the authors. Licensee MDPI, Basel, Switzerland. This article is an open access article distributed under the terms and conditions of the Creative Commons Attribution (CC BY) license (http://creativecommons.org/licenses/by/4.0/). 